\title{
TMD effects in SIDIS at COMPASS
}

\section{Andrea Bressan* on behalf of the COMPASS Collaboration}

University of Trieste and Trieste Division of INFN

E-mail: Andrea.Bressan@ts.infn.it

The COMPASS Experiment at CERN is celebrating this year the $20^{\text {th }}$ anniversary of its' activity after the approval in 1997 and in this presentation an overview of the recent results on the Transverse Momentum Dependent (TMD) effects in semi-inclusive DIS reactions will be given. TMD effects have been investigated both in unpolarised SIDIS by measuring the dependence of charged hadrons multiplicities from the transverse momentum $P_{\mathrm{hT}}$ and by measuring the azimuthal $\cos \phi$ and $\cos 2 \phi$ modulations related to Cahn and to the Boer-Mulders TMD PDF, in transversely polarised SIDIS by measuring the Collins, the Sivers and all the others transverse spin dependent asymmetries, and recently also in the first ever polarized Drell-Yan experiment, started in 2015. Future plans for the investigations of TMDs both in COMPASS will be also briefly presented.

QCD Evolution 2017

22-26 May, 2017

Jefferson Lab Newport News, VA - USA

${ }^{*}$ Speaker. 
The COMPASS experiment was proposed in 1996 as a "COmmon Muon and Proton Apparatus for Structure and Spectroscopy" to study both the hadron structure and address open questions in hadron spectroscopy. After 15 years of data taking many results have been obtained, covering various topics of the hadron structure, namely the longitudinal spin structure of the nucleon in semiinclusive deeply inelastic scattering (SIDIS), the transverse spin structure of the nucleon in SIDIS, in exclusive reactions, and in Drell-Yan scattering, and the hadron multiplicities in unpolarised SIDIS. This short review will be focused on accessing TMDs with SIDIS using both polarised and unpolarised targets. The recent results on weighted Sivers asymmetries will also given, before concluding by mentioning the future measurements we are planning.

\section{The TMD-PDFs}

The leading-twist description of the nuclear structure which takes into account the parton transverse momentum $k_{\perp}$ together with the fraction of longitudinal momentum given by the Bjorken $x$ requires eight transverse momentum dependent parton distribution functions TMD-PDFs. After integration over the transverse momentum three of them reduce to the "collinear" PDFs, namely the number density $f_{1}$, the helicity $g_{1}$ and the transversity distributions $h_{1}$. The other five (i.e. the Boer-Multers $h_{1}^{\perp}$, the Kotzinian-Mulders $h_{1 L}^{\perp}$, the Sivers $f_{1 T}^{\perp}$, the pretzelosity $h_{1 T}^{\perp}$, the worm-gear $g_{1 T}^{\perp}$ ) vanish when integrating over $k_{\perp}$, and their measurement provides important information on the dynamics of the partons in the transverse plane in momentum space [1-3]. They are all independent functions, although, possible relationships can be found depending on model-dependent assumptions.

Particularly interesting is the measurement of the SIDIS cross-section when the target nucleon is transversely polarised. In this case eight (five in case of unpolarised lepton beam) spin-dependent azimuthal modulations of the produced hadrons are expected. All these target spin dependent azimuthal modulations have been investigated by the HERMES and COMPASS Collaborations in pioneering experiments. In particular, HERMES [4,5] and COMPASS [6-8] are up to now the only SIDIS experiments that have shown that the Sivers function [9], the transversity function and the Collins function [10] are different from zero. Out of the eight possible transverse spin azimuthal modulations only the Collins and the Sivers asymmetries differ from zero, while the others, even if hints of signals are present, are all compatible with zero with the present experimental precision. Moreover there is no clear experimental evidence that the second T-odd TMD PDF, the BoerMulders function, which should show up in the azimuthal modulations of the unpolarised SIDIS cross-section, will be different from zero. Finally non-zero results for the asymmetries have been obtained scattering leptons on a proton target, while the corresponding asymmetries on a deuteron target (as measured by COMPASS) are compatible with zero [11,12], hinting at a cancellation between the $u$ - and $d$ - quarks PDFs.

Another very interesting aspect of TMD-PDFs is the peculiar dependence on $Q^{2}$ or their evolution. TMD evolution [13-15] in fact differs from the well-established collinear DGLAP evolution and encloses non-perturbative coefficients which can only be extracted from the data themselves, and as such requires results extracted at very different $Q^{2}$. A definitive answer on this aspect will therefore need very precise data spanning order of magnitude in $Q^{2}$, something that only a dedi- 
cated machine such as an high energy, high intensity polarised electron ion collider will be able to provide.

\section{Unpolarised distribution and modulations}

The study of the dependence of hadron multiplicities on hadron transverse momentum $\vec{P}_{\mathrm{hT}}$ (with respect to the virtual photon) allow to access information on the unpolarised TMD PDF $f_{1}\left(x, k_{\perp}\right)$, convoluted with TMD FF $D_{1}\left(z, p_{\perp}\right)$. Up to order $k_{\perp} / Q$, momentum conservation gives $\vec{P}_{\mathrm{hT}}=z \vec{k}_{\perp}+\vec{p}_{\perp}$ and the structure function $F_{U U}$ is given by the convolution integral $C\left[f_{1} D_{1}\right]$, or:

$$
F_{U U}\left(P_{\mathrm{hT}}\right)=x \sum_{q} e_{q}^{2} \int \delta^{2}\left(\vec{p}_{\perp}-z \vec{k}_{\perp}-\vec{P}_{\mathrm{hT}}\right) f_{1}\left(x, k_{\perp}\right) D_{1}\left(z, p_{\perp}\right) d \mathbf{p}_{\perp}^{2} d \mathbf{k}_{\perp}^{2}
$$

COMPASS has recently send for publication the analysis of the hadron multiplicities from the 2006 ${ }^{6} \mathrm{LiD}$ data [16], covering a broader $P_{\mathrm{hT}}$ range and showing the need to introduce more complex curves than a single Gaussian to fit the data (see Fig. 1), and important kinematic dependencies of the slope of the multiplicities. Data on a liquid hydrogen target have been collected in 2016 and 2017, carrying on the program described in the approved phase-II proposal [17].
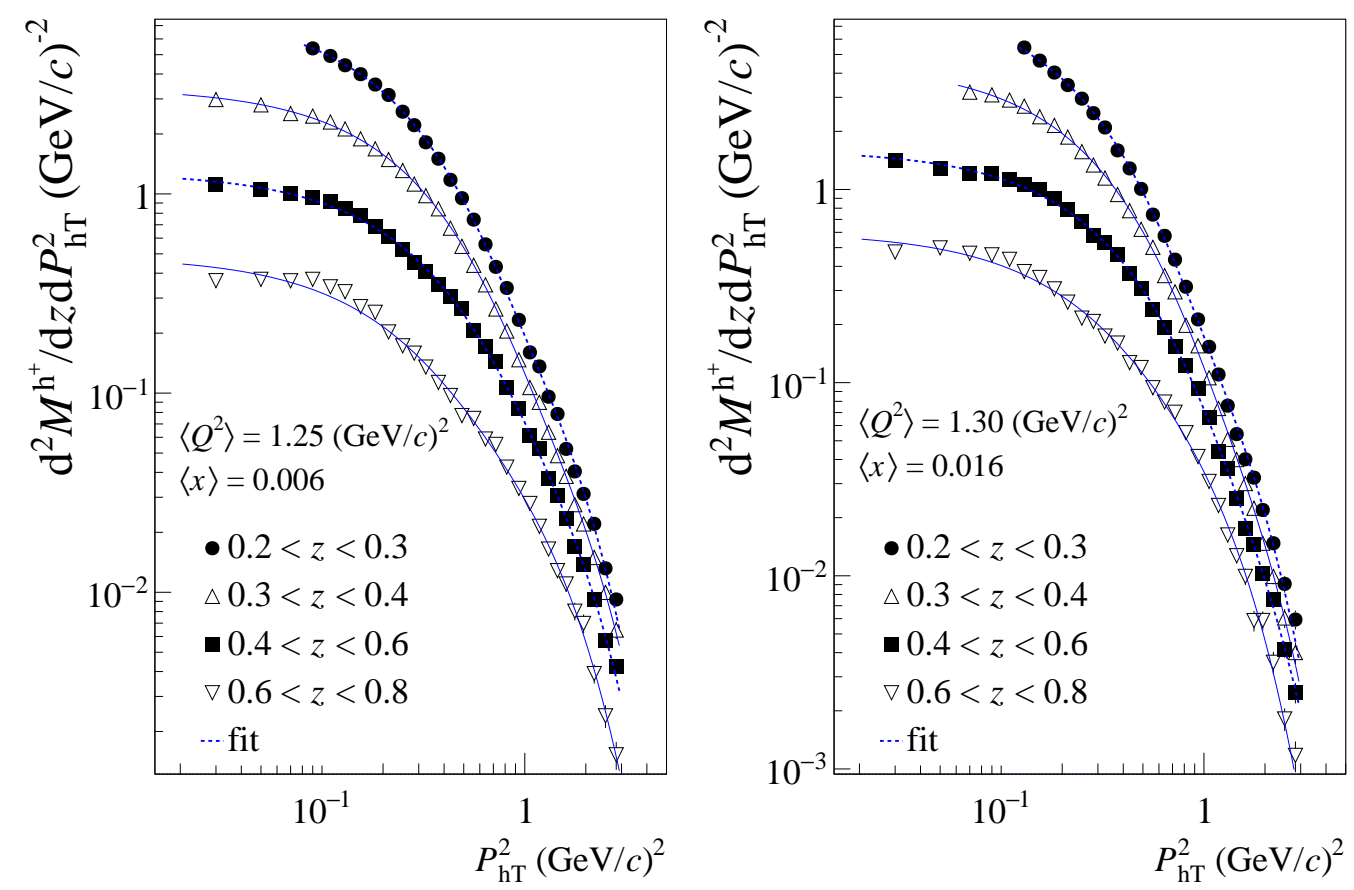

Figure 1: (a) Multiplicities of positively charged hadrons as a function of $P_{\mathrm{hT}}^{2}$ in four $z$ bins at $\left\langle Q^{2}\right\rangle=1.25$ $(\mathrm{GeV} / c)^{2}$ for $\langle x\rangle=0.006$. The curves correspond to the fits using the sum of two exponentials. Only statistical uncertainties are shown and used in the fit. (b) Same as (a) for $\langle x\rangle=0.016$.

Cosine modulations in the azimuthal dependencies of the distribution of the produced hadrons about the direction of the virtual photon can be non-vanishing due to simple kinematic effects (Cahn effect) [18,19], while the interplay between the parton transverse momentum and spin (BoerMulders effect [20]) can generate a leading-twist contribution to the $\cos 2 \phi$ modulations: 


$$
F_{U U}^{\cos 2 \phi}=C\left[-\frac{2\left(\hat{\mathbf{h}} \cdot \vec{k}_{\perp}\right)\left(\hat{\mathbf{h}} \cdot \vec{k}_{\perp}\right)-\vec{k}_{\perp} \cdot \vec{p}_{\perp}}{M M_{h}} h_{1}^{\perp} H_{1}^{\perp}\right]
$$

Perturbative QCD effects, like gluon radiation, can also lead to azimuthal dependencies in the semi-inclusive DIS cross section. However, they contribute mainly at large values of $P_{\mathrm{hT}}$, and are next-to-leading order in the strong coupling constant.

Neglecting twist- 3 contributions only two terms remain:

$$
F_{U U}^{\cos \phi} \simeq-\frac{2 M}{Q} C\left[\frac{\hat{\mathbf{h}} \cdot \vec{p}_{\perp}}{M} f_{1} D_{1}+\frac{\hat{\mathbf{h}} \cdot \vec{k}_{\perp}}{M_{h}} h_{1}^{\perp} H_{1}^{\perp}\right]
$$

where the first (second) term is related to the Cahn (Boer-Mulders) effect. There are no contributions to $F_{U U}^{\cos 2 \phi}$ suppressed as $1 / Q$ however a contribution suppressed as $1 / Q^{2}$ is expected from the Cahn effect to $F_{U U}^{\cos 2 \phi}$. Other contributions beyond $1 / Q$ have not been calculated yet.

At COMPASS, positive and negative hadrons produced by the $160 \mathrm{GeV} / \mathrm{c}$ muon beam scattering off a ${ }^{6} \mathrm{LiD}$ target have been measured in a three-dimensional grid of the relevant kinematic variables Bjorken- $x$, the fraction of the virtual photon energy carried by the hadron $z=E_{\mathrm{h}} / E_{\gamma^{*}}$ and $P_{\mathrm{hT}}$, the hadron transverse momentum with respect to the virtual photon (see Ref. [21]). The data confirm the existence of a sizable $\cos \phi$, showing strong kinematic dependencies, and a not-zero and positive $\cos 2 \phi$ modulations. $A_{U U}^{\cos 2 \phi_{h}}$ shows a relevant dependence on the $x, z$ and $P_{\mathrm{hT}}$ variables, as shown in Fig. 2. The large positive asymmetry values in the low- $x$ region are mainly observed at small $P_{\mathrm{hT}}$ values and large $z$ values. For $P_{\mathrm{hT}}>0.5 \mathrm{GeV} / c A_{U U}^{\cos 2 \phi_{h}}$ becomes smaller and shows a different $x$ dependence.

\section{The Sivers asymmetries}

The Sivers effect was experimentally observed in SIDIS on transversely polarised proton targets, first by the HERMES Collaboration [4,5] and then by the COMPASS Collaboration [7,22], while the COMPASS measurements on a transversely polarised deuteron target $[11,12]$ gave asymmetries compatible with zero. Combined analysis of the proton and deuteron data soon allowed for first extractions of the Sivers function for $u$ - and $d$-quarks [23-26], which turned out to be different from zero, with similar strength and opposite sign, a most important result in TMD physics.

At leading twist the Sivers asymmetry can be written as

$$
A_{S i v}\left(x, z, Q^{2}\right)=\frac{\sum_{q} e_{q}^{2} x f_{1 T}^{\perp q}\left(x, Q^{2}\right) \otimes D_{1}^{q}\left(z, Q^{2}\right)}{\sum_{q} e_{q}^{2} x f_{1}^{q}\left(x, Q^{2}\right) D_{1}^{q}\left(z, Q^{2}\right)}
$$

where $\otimes$ is meant for the convolution $C\left[f_{1 T} D_{1}\right]=\int \delta^{2}\left(\vec{p}_{\perp}-z \vec{k}_{\perp}-\vec{P}_{\mathrm{hT}}\right) f_{1 T}\left(x, k_{\perp}\right) D_{1}\left(z, p_{\perp}\right) d \mathbf{p}_{\perp}^{2} d \mathbf{k}_{\perp}^{2}$ over the transverse momenta of the Sivers function $f_{1 T}^{\perp}$ and of the fragmentation function $D_{1}$. All analyses assume therefore some functional form (in most of the cases Gaussian) for the transverse momentum dependence of $f_{1 T}$ and of the fragmentation functions $D_{1}$.

A way to avoid such assumptions was put forward already twenty years ago [20,27,28]. This was obtained by weighting each hadron entering the asymmetry measurement using as weight the 


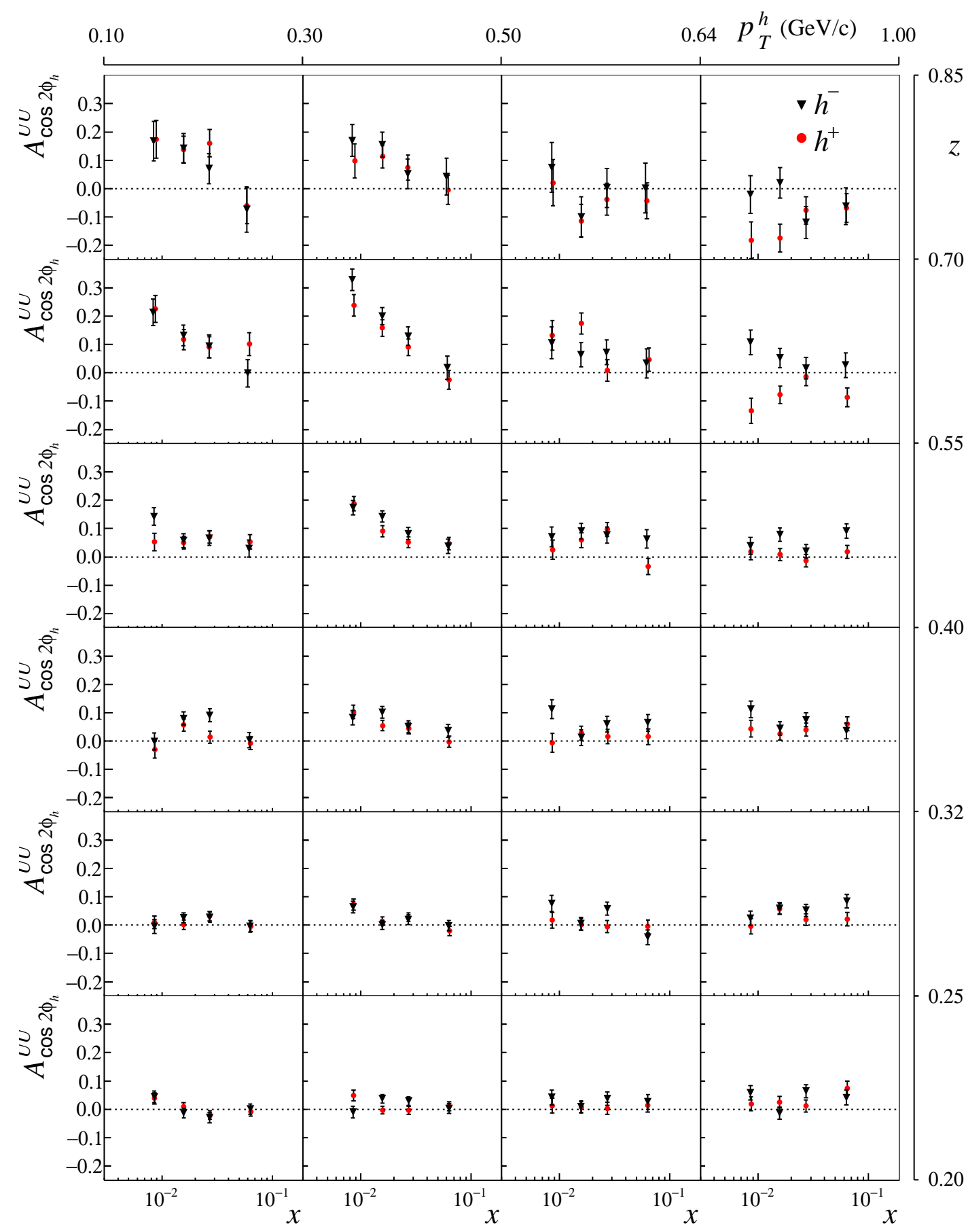

Figure 2: $A_{U U}^{\cos 2 \phi_{h}}$ asymmetries for positive (red points) and negative (black triangles) hadrons as a function of $x$ for the different bins in $P_{\mathrm{hT}}$ ( $p_{T}^{h}$ in the figure, from left to right) and $z$ (from bottom to top).The error bars show statistical uncertainties only. 
measured its transverse momentum $P_{\mathrm{hT}}$ and the energy fraction $z$. Due to the difficulties to evaluate the systematic uncertainties that can be introduced by the experimental acceptances, this method was not adopted by the first analysis of COMPASS and HERMES (a still preliminary results was in any case produced by HERMES [29]). Today with the full knowledge of the apparatus acceptance and following the renewed interest to the weighted asymmetries (see, e.g. [30]), COMPASS has produced new results [31] on the weighted Sivers asymmetries from the data collected in 2010 with the transversely polarised proton target, using $w=P_{\mathrm{hT}} /(z M)$ and $w^{\prime}=P_{\mathrm{hT}} / M$ as weights; only the first will be presented here. With $P_{\mathrm{hT}} /(z M)$ inserted in the convolution integral (i.e. $C\left[w f_{1 T} D_{1}\right]$ ) and with some simple algebra the numerator of Eq. (3.1) becomes the product of the first transverse moment of the Sivers function $f_{1 T}^{\perp(1)}(x)=\int d \mathbf{k}_{\perp}^{2} k_{\perp}^{2} f_{1 T}^{\perp}(x) / M^{2}$ and the fragmentation function $D_{1}$ :

$$
A_{S i v}^{w}(x, z)=2 \frac{\sum_{q} e_{q}^{2} x f_{1 T}^{\perp(1) q}(x) D_{1}^{q}(z)}{\sum_{q} e_{q}^{2} x f_{1}^{q}(x) D_{1}^{q}(z)} .
$$

Moreover assuming $u$-dominance for positive hadrons production on protons, the fragmentation function cancels out and the asymmetry simply becomes

$$
A_{S i v}^{w}(x, z) \simeq 2 \frac{f_{1 T}^{\perp(1) u}(x)}{f_{1}^{u}(x)} .
$$

which allows a simple and direct extraction of the first moment $f_{1 T}^{\perp(1) u}$. Fig. 3 (left) shows the $P_{\mathrm{hT}} /(z M)$ weighted asymmetries for positive hadrons while Fig. 3 (right) is for negative hadrons, together with the already published asymmetries [7]. As expected, the trend of the weighted asymmetries 'follows' both for positive and negative hadrons the one of the standard Sivers asymmetries. The results for positive hadrons at large $x$, where $\left\langle Q^{2}\right\rangle$ reaches $\sim 20(\mathrm{GeV} / c)^{2}$, constitute the first direct measurement of $f_{1 T}^{\perp(1) u}(x) / f_{1}^{u}(x)$. Given the similarity of the trend of the weighted asymmetries $A_{S i v}^{w}$ to the standard asymmetries $A_{S i v}$, the ratios $R^{w}=A_{S i v}^{w} / A_{S i v}$ as also been evaluated; for positive hadrons the values of $R^{w}$ are given in Fig. 4. In spite of the large statistical uncertainties the ratios are compatible with a constant value, which is rather well determined (1.6 \pm 0.1$)$. Also, the values in the different $x$ bins agree rather well with those of $\left\langle P_{\mathrm{hT}} /(z M)\right\rangle$, as expected.
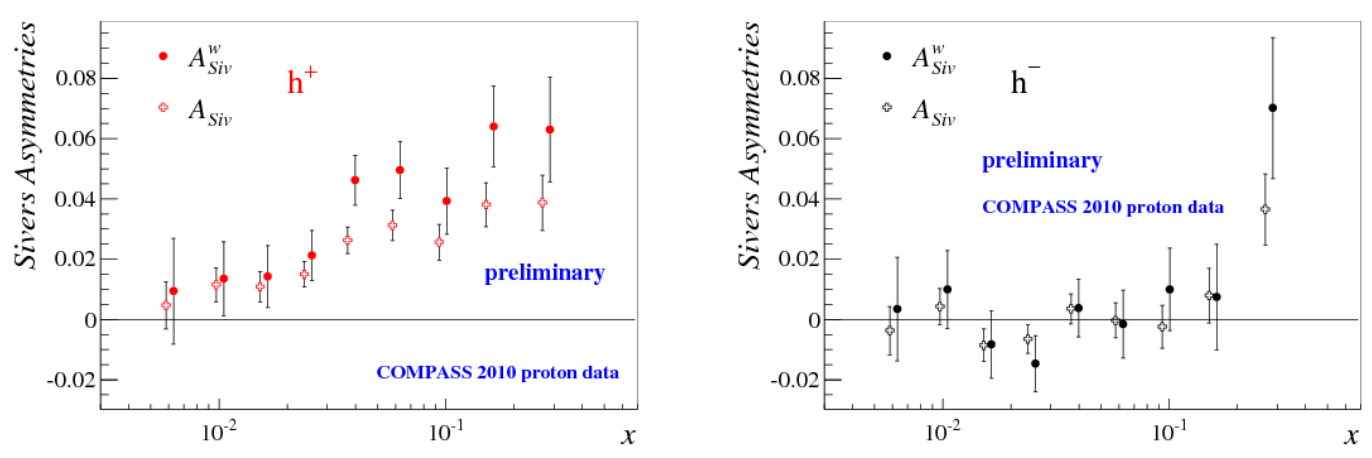

Figure 3: Full points: $A_{S i v}^{w}$ in the nine $x$ bins for positive (left panel) and negative (right panel) hadrons. The open crosses are the standard Sivers asymmetries $A_{S i v}$ from Ref. [7]. 


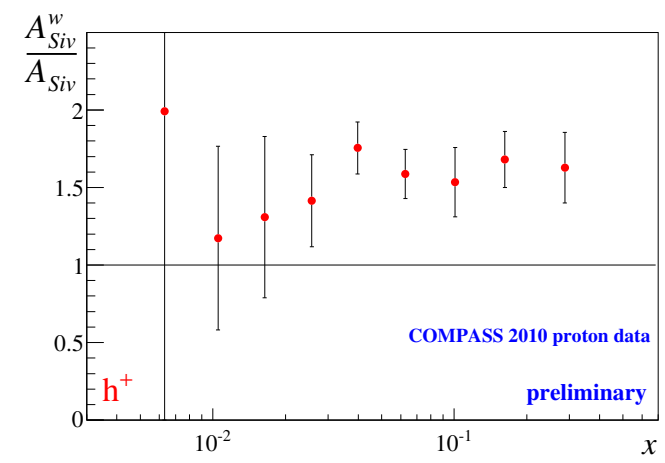

Figure 4: The ratios $R^{w}$ between $A_{S i v}^{w}$ and $A_{S i v}$ in the nine $x$-bins for positive hadrons.

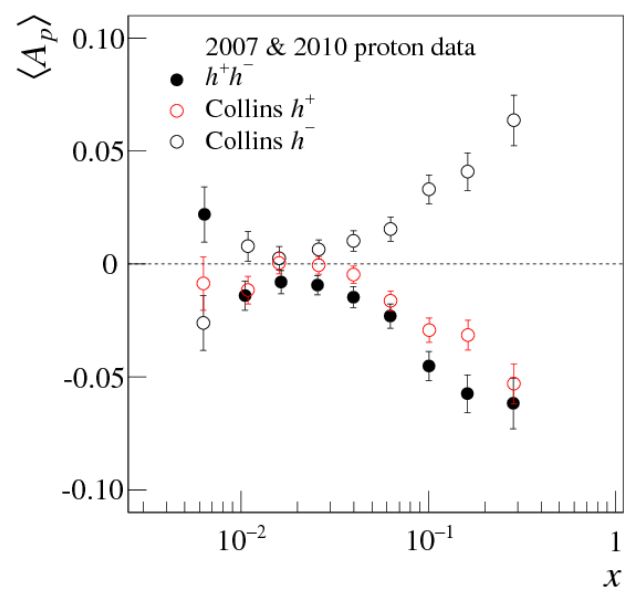

Figure 5: Comparison of the di-hadron asymmetry with the Collins asymmetry from the combined 2007 and 2010 proton data [8].

\section{The transversity PDF}

COMPASS measurements of transversity related asymmetries have been central for the programme with the transversely polarised target, and will not be reviewed here. As a glimpse of this only some surprising but very interesting result will be shown in Fig. 5, where the Collins asymmetries for positive and negative hadrons are compared to the di-hadron asymmetry, namely to the azimuthal modulation of the plane containing two oppositely charged hadrons. This di-hadron asymmetry can be expressed as the product of the quark transversity distribution and a chiral-odd di-hadron fragmentation function, which survives after integration over the two hadron momenta, and thus can be analyzed in the framework of collinear factorization. The mirror symmetry of the Collins asymmetry of positive and negative hadrons and the similarity between the positive hadron Collins asymmetry and the di-hadron asymmetry is intriguing. An in-depth comparison between the two asymmetries [32] suggests that both the single hadron and the di-hadron transverse-spin dependent fragmentation functions are driven by the same elementary mechanism. This mechanism is very well described trough the ${ }^{3} P_{0}$ recursive string fragmentation model [33], an approach that can be also used to generate spin dependent hadronization [34]. 

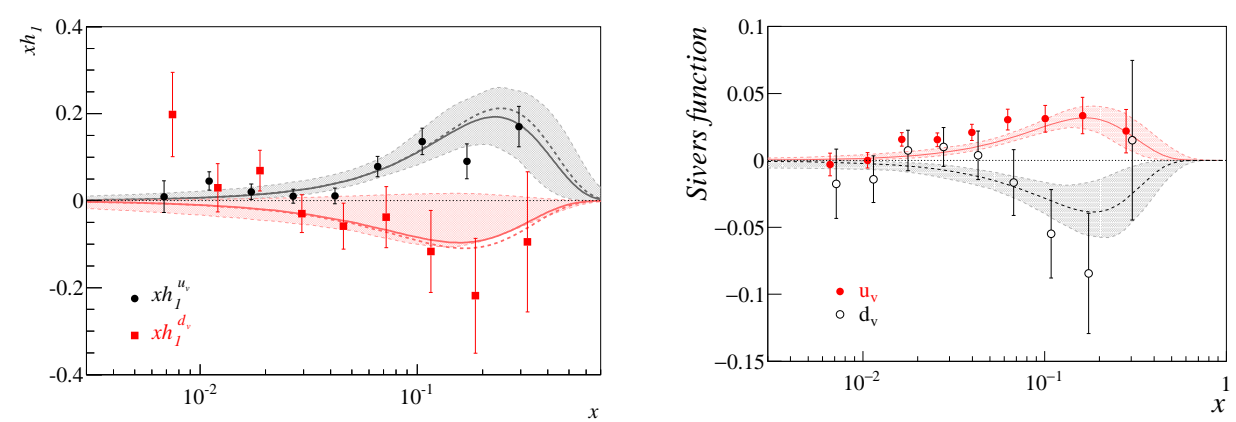

Figure 6: The transversity and Sivers PDFs extracted point-by-point using the existing COMPASS $\mathrm{p}$ and $\mathrm{d}$ data from Ref. [36,37]. The curves are the results of fits to the COMPASS and HERMES data and, for transversity, to the Belle data. Note that the uncertainty band for the $d$-quark transversity is reduced by imposing the Soffer bound.

\section{Future SIDIS measurements}

Very recently the COMPASS Collaboration has submitted to CERN a proposal for an extension of the present programme to perform two measurements: one year of SIDIS on a transversely polarised deuteron target and one year of elastic muon proton scattering [35]. The need to collect more data with a transversely polarised deuteron target is made clear by looking to Fig. 6 showing the point-by-point extraction of the $u$ - and $d$ - transversity PDF of Ref. [36]. The limited precision in the extraction of $h_{1}^{d}$ from the present data sample is evident.

The statistical accuracy that can be obtained by integrating the same luminosity on target of the 2010 run is given in Fig. 7, which shows both the values of transversity (open points) extracted from the existing $\mathrm{p}$ and $\mathrm{d}$ data, and the corresponding error bars (closed points) estimated using the existing $\mathrm{p}$ data and the $\mathrm{d}$ data, with the projected errors of the new measurement. The gain in precision for the $d$-quark ranges from a factor of 2 at small $x$ to a factor of 5 at large $x$, and is also important for the $u$-quark.

If this proposal will be approved, as we hope, by CERN, the new data will provide large $Q^{2}$ results in the $x$-range covered by JLab12, which is very important to evaluate the size of the $Q^{2}$ evolution, and will provide lower- $x$ data (down to $x=0.003$ ) which are essential both to perform the integrals necessary to evaluate the tensor charges and to estimate the TMD PDFs of the sea quarks. The data will moreover remain unique until the planned Electron Ion Collider (EIC) [38] will be on the floor.

\section{References}

[1] P.J. Mulders and J. Rodrigues, Phys. Rev. D63 (2001) 094021, hep-ph / 0009343.

[2] A. Bacchetta et al., JHEP 02 (2007) 093, hep-ph/ 0611265.

[3] C. Lorce and B. Pasquini, Phys.Rev. D84 (2011) 034039, arXiv:1104. 5651.

[4] HERMES Collaboration, A. Airapetian et al., Phys. Rev. Lett. 94 (2005) 012002, hep-ex/0408013. 


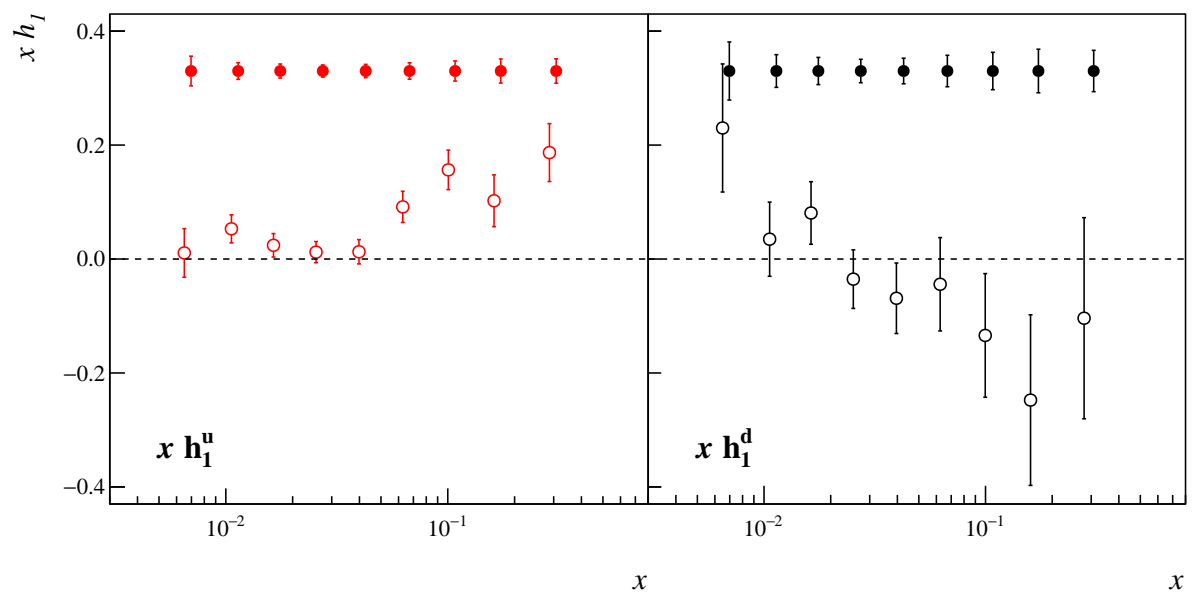

Figure 7: Values of $u_{v}$-quark (left) and $d_{v}$-quark (right) transversity extracted from the existing p and d data (open points ), and the corresponding error bars estimated using the existing $\mathrm{p}$ data and the new $\mathrm{d}$ data (closed points).

[5] HERMES Collaboration, A. Airapetian et al., Phys. Rev. Lett. 103 (2009) 152002, arXiv:0906.3918.

[6] COMPASS Collaboration, C. Adolph et al., Phys.Lett. B717 (2012) 376, arXiv: 1205.5121.

[7] COMPASS Collaboration, C. Adolph et al., Phys.Lett. B717 (2012) 383, arXiv: 1205.5122.

[8] COMPASS Collaboration, C. Adolph et al., Phys. Lett. B736 (2014) 124, arXiv: 1401.7873.

[9] D.W. Sivers, Phys. Rev. D41 (1990) 83.

[10] J.C. Collins, Nucl. Phys. B396 (1993) 161, hep-ph/ 9208213.

[11] COMPASS Collaboration, V.Y. Alexakhin et al., Phys. Rev. Lett. 94 (2005) 202002, hep-ex/0503002.

[12] COMPASS Collaboration, E.S. Ageev et al., Nucl. Phys. B765 (2007) 31, hep-ex/ 0610068.

[13] J. Collins, Foundations of perturbative QCD (Cambridge University Press, 2011).

[14] M.G. Echevarria et al., Eur.Phys.J. C73 (2013) 2636, arXiv: 1208.1281.

[15] S.M. Aybat, A. Prokudin and T.C. Rogers, Phys.Rev.Lett. 108 (2012) 242003, arXiv: 1112.4423.

[16] COMPASS Collaboration, M. Aghasyan et al., (2017), arXiv:1709.07374.

[17] COMPASS Collaboration, F. Gautheron et al., (2010), COMPASS-II Proposal.

[18] R.N. Cahn, Phys. Lett. B78 (1978) 269.

[19] R.N. Cahn, Phys. Rev. D40 (1989) 3107.

[20] D. Boer and P.J. Mulders, Phys. Rev. D57 (1998) 5780, arXiv: hep-ph/ 9711485.

[21] COMPASS Collaboration, C. Adolph et al., Nucl. Phys. B886 (2014) 1046, arXiv:1401. 6284.

[22] COMPASS Collaboration, M.G. Alekseev et al., Phys. Lett. B692 (2010) 240, arXiv: 1005.5609.

[23] A.V. Efremov, K. Goeke and P. Schweitzer, Phys. Lett. B568 (2003) 63, hep-ph/ 0303062. 
[24] W. Vogelsang and F. Yuan, Phys. Rev. D72 (2005) 054028, hep-ph / 0507266.

[25] J.C. Collins et al., Phys. Rev. D73 (2006) 014021, hep-ph/ 0509076.

[26] M. Anselmino, M. Boglione and S. Melis, Phys.Rev. D86 (2012) 014028, arXiv:1204.1239.

[27] A.M. Kotzinian and P.J. Mulders, Phys. Rev. D54 (1996) 1229, hep-ph/ 9511420.

[28] A.M. Kotzinian and P.J. Mulders, Phys. Lett. B406 (1997) 373, arXiv: hep-ph / 9701330.

[29] HERMES Collaboration, I.M. Gregor, Acta Phys. Polon. B36 (2005) 209.

[30] Z.B. Kang, I. Vitev and H. Xing, Phys.Rev. D87 (2013) 034024, 1212 . 1221.

[31] COMPASS Collaboration, F. Bradamante, 22nd International Symposium on Spin Physics (SPIN 2016) Urbana, IL, USA, September 25-30, 2016, 2017, 1702.00621.

[32] COMPASS Collaboration, C. Adolph et al., Phys. Lett. B753 (2016) 406, arXiv: 1507 . 07593.

[33] X. Artru, (2002), arXiv:0207309.

[34] A. Kerbizi et al., 22nd International Symposium on Spin Physics (SPIN 2016) Urbana, IL, USA, September 25-30, 2016, 2017, 1701.08543.

[35] COMPASS Collaboration, J. Friedrich et al., (2017), Addendum to the COMPASS-II Proposal.

[36] A. Martin, F. Bradamante and V. Barone, Phys. Rev. D91 (2015) 014034, arXiv: 1412.5946.

[37] A. Martin, F. Bradamante and V. Barone, Phys. Rev. D95 (2017) 094024, arXiv: 1701.08283.

[38] A. Accardi et al., Eur. Phys. J. A52 (2016) 268, arXiv:1212 .1701. 